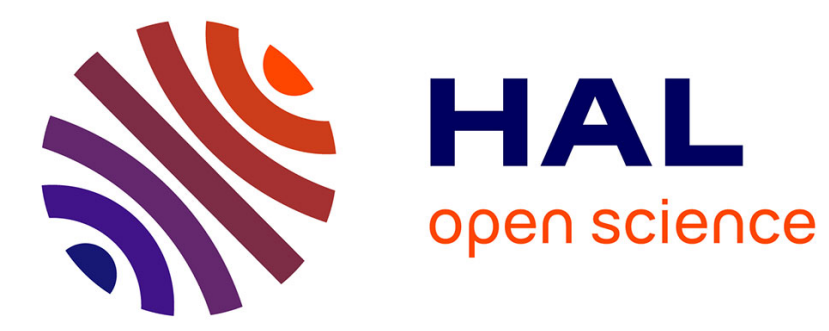

\title{
3D statistical models for tooth surface reconstruction
}

\author{
Stéphanie Buchaillard, S. H. Ong, Yohan Payan, Kelvin Foong
}

\section{To cite this version:}

Stéphanie Buchaillard, S. H. Ong, Yohan Payan, Kelvin Foong. 3D statistical models for tooth surface reconstruction. Computers in Biology and Medicine, 2007, 37 (10), pp.1461-71. 10.1016/j.compbiomed.2007.01.003 . hal-00169766

\section{HAL Id: hal-00169766 https://hal.science/hal-00169766}

Submitted on 4 Sep 2007

HAL is a multi-disciplinary open access archive for the deposit and dissemination of scientific research documents, whether they are published or not. The documents may come from teaching and research institutions in France or abroad, or from public or private research centers.
L'archive ouverte pluridisciplinaire HAL, est destinée au dépôt et à la diffusion de documents scientifiques de niveau recherche, publiés ou non, émanant des établissements d'enseignement et de recherche français ou étrangers, des laboratoires publics ou privés. 


\title{
3D Statistical Models for Tooth Surface Reconstruction
}

\author{
${ }^{1}$ Stéphanie I. Buchaillard, ${ }^{1,2}$ S. H. Ong, ${ }^{3}$ Yohan Payan and ${ }^{4}$ Kelvin Foong \\ ${ }^{1}$ Dept of Electrical and Computer Engineering, National University of Singapore, Singapore \\ ${ }^{2}$ Division of Bioengineering, National University of Singapore, Singapore \\ ${ }^{3}$ TIMC-IMAG Laboratory, UMR CNRS 5525, Faculty of Medicine, France \\ ${ }^{4}$ Department of Preventive Dentistry, National University of Singapore, Singapore
}

\begin{abstract}
This paper presents a method to reconstruct the 3D surface of a tooth given partial information about its shape. A statistical model comprising a mean shape and a series of deformation modes is obtained offline using a set of specimens. During reconstruction, rigid registration is performed to align the mean shape with the target. The mean shape is then deformed to approximate the target by minimizing the sum of squared distances between the two surfaces according to the deformation modes. The method is shown to be efficient for the recovery of tooth shape given crown information.
\end{abstract}

\section{INTRODUCTION}

7 HE application of visualization techniques to dentistry has experienced a rapid growth and now includes educational displays, training for delicate procedures, treatment simulations and even communication tools with patients. Presurgery simulation systems have proven to be useful in the treatment of malocclusion [1], [2], identifying the optimal cutting plane for an implant [3] and selecting an optimal operative method [4]. Visualization is an important component in devices for computed-aided surgery [5]. Finally, computerized video imaging techniques are now widely used by dentists to discuss the outcome of orthognathic surgery with their patients [6].

Many dental and maxillofacial surgery applications such as endodontic procedures, treatment of malocclusion problems, and treatment simulations require an accurate knowledge of 
the $3 \mathrm{D}$ shape of teeth and the positions of the tooth roots. For example, orthodontists can reposition the teeth in cases of malocclusion (improper positioning of the teeth and jaws) by using brackets. In this situation, knowing the exact location, orientation and 3D shape of the teeth would assist the clinician in planning appropriate movements during treatment. Having a good knowledge of the shape of a given tooth is also extremely helpful in creating implants. Currently, the shape of a tooth in the mouth is represented in two dimensions in an X-ray film. Since teeth are 3D structures with complex shapes, an accurate 3D representation of tooth shape is vital in facilitating clinical treatment. Teeth are also commonly used in forensic medicine for identification purposes [7]. They are the most durable parts of the body but, often, only fragments are available and the missing part needs to be estimated.

Computer tomography (CT) is effective in obtaining 3D data for the applications mentioned above. However, CT imaging of dental patients is usually not indicated, as this imaging modality is radiologically invasive and of relatively low resolution. Consequently, rather than working with CT data, orthodontists regularly employ plaster casts of the patient's dentition, also known as study models. These plaster models are used to prepare treatment plans and for making accurate measurements. However, they only provide information about the crowns of the teeth but none of the roots, which are hidden in the gum. A solution is to fit a tooth virtually onto the dental cast to obtain an estimate of root position and orientation (Fig. 1).

To overcome the lack of original 3D data, alternative methods are necessary to obtain tooth shape when the information is simply missing, or to avoid using radiologically invasive methods that are not clinically justified. Different methods exist for reconstructing 3D shape using 2D information [8]. Enciso et al. [9], for example, propose a 3D reconstruction based on a patient's $2 \mathrm{D}$ radiograph, producing a "best fit" patient-specific 3D geometric polygonal mesh of a tooth using thin-plate splines. A significant limitation of this method is that it does not take into account the possible shape variations of a given tooth and uses only 2D data. 
Other methods exploit sparse 3D data [10] to represent fine details.

The above approaches require a good knowledge of the global 3D shape of the object of interest, which we do not possess. Given only partial 3D information (e.g., the tooth crown or the tooth root), our objective is to obtain a good estimate of the entire tooth shape without the use of X-ray or any other imaging modality. The technique developed by Fleute et al. [11] provides the basic idea for such a reconstruction but in its original form is not suitable for high-resolution data. In our application, significant improvements in the accuracy and speed of shape recovery are possible through the use of high-resolution models, better control on the input volume, and a more efficient optimization method. High-resolution crown models may be obtained, for example, by means of a laser scan of a dental study model and performing a segmentation, as described by Kondo et al. [12]. Once a 3D crown model has been built, we would still require a method to reconstruct the root in order to obtain a better knowledge of tooth shape and orientation, which would consequently facilitate treatment and improve the quality of the clinical outcome.

This paper is organized as follows. Section II presents the construction of a statistical shape model whose variations describe the main ways in which a particular tooth can vary. Section III is devoted to the registration problem and explains how the statistical model can be fitted to the patient's crown to provide a good estimate of the shape and size of the reconstructed tooth. In Section IV, experimental results are presented to demonstrate the capabilities of this approach. The paper ends with the conclusion in Section V.

\section{Construction of a Generic Surface Model}

\section{A. Data Collection}

Twenty-two exemplars of the second upper right premolar were scanned with a SkyScan1076 micro-CT scanner at a resolution of $35 \mu \mathrm{m}$ to give reconstructed specimens with up to 72,000 points and 150,000 triangles. The contour of every tooth was manually extracted on 
each slice of the micro-CT and the resulting teeth subsampled to a new resolution of $105 \mu \mathrm{m}$. A generic tooth model was then matched to each 3D exemplar model (using the procedure described by Szeliski and Lavallée [13]) to obtain a point-to-point correspondence between the different specimens, resulting in a collection of $N=223 \mathrm{D}$ training shapes of the same tooth. This approach requires aligning manually each training tooth with a generic tooth that is in the format of a 3D triangular mesh of $M$ points. Nonrigid registration [13] using free-form deformation [14] and splines is performed hierarchically to deform the generic tooth to match each training tooth. Each resulting example is finally represented by a vector $\mathbf{m}=\left(x_{0}, y_{0}, z_{0}, \ldots, x_{M-1}, y_{M-1}, z_{M-1}\right)$.

Fig. 2(a) shows the generic tooth model used for matching. One of the exemplar models reconstructed from microtomography is seen in Fig. 2(b). Fig. 2(c) shows the result of the nonrigid registration of the generic tooth model to this exemplar. The minimum, maximum and root-mean-squared (RMS) Hausdorff distance (HD) between the 3D tooth model reconstructed from microtomography and the model after matching was computed to evaluate the performance of the point-to-point correspondence process. The results, in Table I, show that the matching process leads globally to highly satisfactory results.

Table I: Hausdorff distance of the point-to-point correspondence matching process.

\begin{tabular}{l|lll|ccc}
\hline & \multicolumn{3}{|c|}{ HD $(\mathrm{mm})$} & \multicolumn{3}{c}{ HD (\% teeth height) } \\
& Max & Mean & RMS & Max & Mean & RMS \\
\hline Minimum & 0.55 & 0.06 & 0.08 & 2.76 & 0.30 & 0.39 \\
Maximum & 2.61 & 0.26 & 0.39 & 13.00 & 1.33 & 2.03 \\
Mean & 1.07 & 0.12 & 0.18 & 5.48 & 0.61 & 0.91 \\
Variance & 0.2493 & 0.0032 & 0.0080 & 6.3548 & 0.0902 & 0.2188 \\
\hline
\end{tabular}

\section{B. Construction of a Point Distribution Model}

A statistical shape model, the point distribution model (PDM) [15], computed from principal component analysis (PCA) can be used to describe the average shape and shape variations 
of a set of sample models. The mean shape $\overline{\mathbf{m}}$ is defined using:

$$
\overline{\mathbf{m}}=\frac{1}{N} \sum_{i=0}^{N-1} \mathbf{m}_{i}
$$

The modes of variation, representing the ways in which the points tend to move together, can be found by computing the eigenvectors $\mathbf{e}_{i}$ of the covariance matrix $\mathbf{R}_{a c}$ :

$$
\mathbf{R}_{a c}=\frac{1}{N-1} \sum_{i=0}^{N-1}\left(\mathbf{m}_{i}-\overline{\mathbf{m}}\right)\left(\mathbf{m}_{i}-\overline{\mathbf{m}}\right)^{T}
$$

Given the high resolution of the 3D models, a direct estimation of the eigenvectors of $\mathbf{R}_{a c}$ is not feasible. Instead of working in the variable (tooth point) space $\mathbb{R}^{3 \times M}$ (dimension $3 \times M)$, the eigenvectors are defined in training-exemplar space $\mathbb{R}^{N}($ dimension $N<3 \times M)$. Simple mathematical considerations [16] give us an immediate correspondence between the eigenvectors and eigenvalues in these two spaces. The eigenvalues in $\mathbb{R}^{3 \times M}$ and $\mathbb{R}^{N}$ are identical, and if $\left(\lambda_{\alpha}, \mathbf{u}_{\alpha}\right)$ is a 2-tuple (an eigenvector and its associated eigenvalue) in $\mathbb{R}^{3 \times M}$, then $\left(\lambda_{\alpha}, \mathbf{v}_{\alpha}\right)$ is also a similar 2-tuple in $\mathbb{R}^{3 \times N}$, where

$$
\mathbf{v}_{\alpha}=\frac{1}{\sqrt{\lambda_{\alpha}}} \mathbf{X} \mathbf{u}_{\alpha}
$$

and the rows of the matrix $\mathbf{X}$ correspond to the tooth points $(N \times 3 M$ matrix $)$.

The proportion of the total variance accounted for by each vector is equal to the corresponding eigenvalues. Consequently, the eigenvectors $\mathbf{e}_{i}$ associated with the maximum eigenvalues $\lambda_{i}$ correspond to the major deformation modes. Fig. 3(a) shows the eigenvalues of the autocorrelation matrix and Fig. 3(b) the cumulative percentage of variability given the number of components. Seven components can account for $95 \%$ of the variability, while thirteen components explain more than $99 \%$ of the variability between the different samples. Any shape belonging to the training set can be approximated as a sum of the mean model 
and a linear combination of the first $N_{p c}$ modes, i.e.,

$$
\mathbf{m}=\overline{\mathbf{m}}+\sum_{i=0}^{N_{p c}-1} \omega_{i} \mathbf{e}_{i}
$$

where $\omega_{i}$ are the weights associated with the eigenvectors $\mathbf{e}_{i}$. By constraining every $\omega_{i}$ such that $-K_{1, i} \lambda_{i} \leq \omega_{i} \leq K_{2, i} \lambda_{i}$ ( $K_{1, i}$ and $K_{2, i}$ constant values) we can limit the deviations from the mean model.

Fig. 4 shows, respectively, the influence of the first and second modes of the decomposition. In each figure, the tooth in the middle represents the mean shape. The tooth on the left shows the new shape when the first (or second) mode is weighted by a coefficient $-K_{1, i} \lambda_{i}(i=1$ or 2), while the tooth on the right shows the new shape when the first (or second) mode is weighted by a coefficient $+K_{2, i} \lambda_{i}$. The first mode has a huge influence on the size of the teeth and on the global shape of the root. The second has a much smaller influence on the tooth height but a great impact on the width of the root. The other modes have different influences that cannot always be simply described.

\section{3D Mesh Model}

The mean model consists of an unorganized cloud of points. In order to obtain a mesh boundary representation of the patient's tooth after reconstruction, a $3 \mathrm{D}$ reconstruction is performed using a marching cube algorithm [17] after extraction of the isosurface.

\section{TOOTH RECONSTRUCTION}

Once the statistical model has been defined, the patient's tooth shape is computed from the optimal rigid and elastic transformations of the mean model following Fleute et al. [11]. We aim to match the patient's crown or root with the corresponding region of our statistical model by determining the contribution of the different modes of the statistical model. The 
crown (or root) shape variations with respect to the mean model are then used to infer the root (or crown) shape.

\section{A. Initial Registration}

In order to expedite the reconstruction of the target tooth, an intermediate step can be included between PCA (Section II-B) and the final deformation. The iterative closest point (ICP) algorithm [18] can be used to perform determine the best possible alignment between the two models. This step is implemented with manual rigid registration and, if necessary, scaling. Although not mandatory, it can greatly decrease the running time of the reconstruction procedure and improve the final result.

\section{B. Nonrigid Deformation of the Statistical Model}

The optimum parameter values can be computed through the minimization of a merit (or energy) function to measure the goodness-of-fit. The merit function used here (5) is the classical mean-squared distance between the crowns (or roots) of the two volumes:

$$
\begin{array}{r}
E(\mathbf{p})=\sum_{i=0}^{M_{c}-1} \min \left(\left\|\mathbf{d}_{j}-\mathbf{m}_{i}\right\|^{2}\right)_{1 \leq j \leq K} \\
\text { with } \quad \mathbf{m}=\mathbf{R}\left(\overline{\mathbf{m}}+\sum_{l=0}^{N_{p c}-1} \omega_{l} \mathbf{e}_{l}\right)+\mathbf{T}
\end{array}
$$

where $\mathbf{p}$ is a vector representing the different parameters to adjust, $M_{c}$ the number of crown (or root) points of the PDM, $K$ the number of points of the target tooth, $d$ the vector representing the target (dimension $3 \times K$ ), $\mathbf{T}$ a translation vector, $\mathbf{R}$ a rotation matrix, $\mathbf{e}_{l}$ the principal components obtained in Section II-B, and $N_{p c}$ the number of principal components selected.

We have to estimate the six components that define the rigid-body transformation between the two volumes (three parameters for $\mathbf{T}$ and three parameters for $\mathbf{R}$ ) as well as the optimum weights for the $N_{p c}$ principal components, i.e., we have to solve an optimization problem 
having only $6+N_{p c}$ parameters. The optimization of $E(\mathbf{p})$ is performed using the LevenbergMarquardt algorithm [19]. To compute the minimum distances, two different options were tested, one with (Method 1) and the other without (Method 2) the use of a pre-computed distance map.

Computing the minimum distances is the most computationally expensive part of the minimization. In the first case, distances are approximated using an octree-spline distance map [13] [20]. The two volumes (target and deformable models) are enclosed in a bounding box and a classical octree decomposition is realized based on the points of the patient's tooth. Fig. 5 shows an example of an octree decomposition based on the points of a tooth root (cloud of points).

For each corner of the terminal octants, the minimum distance to the patient's tooth is computed and stored. Given a new point $P$, we only need to find the octant the point belongs to in order to determine the minimum distance, followed by realizing a trilinear interpolation over the eight corners of this octant. This method allows us to obtain a good approximation of the required distances. The partial derivatives with respect to every component can be computed by Ridder's method of polynomial extrapolation.

The second method uses $k$ d-trees [21] that can be built in $\mathbf{O}(M \log M)$. A $k$ d-tree is a binary tree used to represent data of dimension $d$ (here $d=3$ ). Each node of the binary tree represents a subset of the data record and a partitioning of that subset. The structure of a $k \mathrm{~d}$-tree makes it very easy to compute the required distances. This second method requires the construction of a new $k$ d-tree after each iteration of the Levenberg-Marquardt algorithm. We can obtain the true distances and a closed-form expression for the gradient of $E(\mathbf{p})$ becomes available.

The optimization process can lead to a local minimum instead of the expected global minimum. To overcome this difficulty, the number of modes can be increased successively 
during the minimization process, beginning with the more significant ones until the $N_{p c}$ modes are included in the minimization or sufficient precision is reached.

\section{EXPERIMENTAL RESULTS AND DISCUSSION}

\section{A. Reconstruction Based on Crown Information}

1) Leave-one-out test: Different experiments were conducted to investigate the validity of the method described in this paper. Here we present the results obtained using crown information only and $N_{p c}=7$ modes of deformation for the statistical model. A leave-oneout test was performed as described below. For each of the $N$ teeth belonging to the training set, the following test is realized:

- PCA is first performed on the $N-1$ other teeth (excluding tooth $T_{i}$ ) and new modes are defined.

- $T_{i}$ 's crown is extracted.

- The crown of the mean shape model is extracted as well as the corresponding deformation modes.

- The new modes are then used to reconstruct $T_{i}$.

- The distance between the tooth $T_{i}$ before (root included) and after reconstruction $\left(\hat{T}_{i}\right)$ is then found.

To estimate the distance between two volumes represented by triangular meshes, we use the main Hausdorff distance described by Garland et al. in [22]. Tables II and III present the results obtained using or not a distance map to compute the minimum distances between the mean shape and the target. Figure 6 shows some examples of shape recovery for three different specimens.

For all the teeth of the test set, the reconstruction processes lead to a perfect match between the crowns of $T_{i}$ and $\hat{T}_{i}$ and in the majority of the reconstructions, a very good approximation of the tooth heights and widths (Fig. 6(a) and Fig. 6(b)). 
Table II: Leave-one-test results: reconstruction based on crown information (Method 1).

\begin{tabular}{l|lll|lll}
\hline & \multicolumn{3}{|c|}{ HD (mm) } & \multicolumn{3}{c}{ HD (\% teeth height) } \\
& Max & Mean & RMS & Max & Mean & RMS \\
\hline Minimum & 1.21 & 0.17 & 0.25 & 6.21 & 0.87 & 1.28 \\
Maximum & 4.22 & 0.50 & 0.91 & 21.66 & 2.57 & 4.67 \\
Mean & 2.10 & 0.29 & 0.49 & 10.77 & 1.49 & 2.51 \\
Variance & 0.7057 & 0.0103 & 0.0306 & 18.5841 & 0.2712 & 0.8058 \\
\hline
\end{tabular}

Table III: Leave-one-test results: reconstruction based on crown information (Method 2).

\begin{tabular}{l|lll|clc}
\hline & \multicolumn{3}{|c|}{ HD (mm) } & \multicolumn{3}{c}{ HD (\% teeth height) } \\
& Max & Mean & RMS & Max & Mean & RMS \\
\hline Minimum & 0.84 & 0.09 & 0.12 & 4.47 & 0.48 & 0.64 \\
Maximum & 3.76 & 0.46 & 0.81 & 19.99 & 2.44 & 4.31 \\
Mean & 2.02 & 0.25 & 0.43 & 10.74 & 1.33 & 2.29 \\
Variance & 0.7018 & 0.0085 & 0.0291 & 19.8542 & 0.2405 & 0.8232 \\
\hline
\end{tabular}

Only one tooth in the training set (Fig. 6(c)) produced results far from those expected using any one of the two methods. Indeed, the reconstruction being based on a statistical representation, outliers will lead to erroneous results. For this particular specimen, the correlation usually observed between the height and width of a tooth and the size (i.e., the shape and width) of the crown is not followed in that the crown is extremely large compared to its height; this gave rise to a reconstructed model that was much longer than the original one.

2) Reconstruction using patient data: A second test was then realized using real data. Though the test realized above is realistic in forensic medicine, the data used by orthodontists usually present losses at the interstices as shown in Fig. 7, whatever the segmentation method used to extract the crown.

A study model was digitized using the Cyberware Rapid 3D Digitizer Model 3030R-HIREZ laser scanner. The upper right second premolar was then extracted using Kondo et al.'s method of segmentation [12] (Fig. 8(a)) and the 3D shape of the tooth determined (Fig. 8(b)). Despite the important loss of information introduced by the segmentation, reconstruction results in an excellent match between the original crown and those of the tooth. The reconstructed tooth was then combined with the orthopantomogram (a panoramic X-ray of the jaw and the teeth) 
of the same patient (Fig. 8(c)) to test the validity of the shape reconstruction. As shown in Fig. 8(d), the method proposed led to a very good estimate of the tooth shape and size.

\section{B. Reconstruction Based on Root Information}

In this section, we present the results obtained using root information only and the seven modes of deformation for the statistical model. This kind of reconstruction finds applications mainly in forensic medicine when reconstructing a dentition for identification. A leave-oneout test using the $N$ specimens was performed as described in Section IV-A.1.

As shown in Tables IV and V and Figure 9, the results are far from reliable. The HD between the original teeth and their reconstructed versions using root information are smaller than that previously obtained (reconstruction using crown information), but larger with respect to the part being inferred (crown or root). The main problem comes from the root shape. Its simplicity, in comparison with the complexity of the molar shape, makes the matching process more difficult. Indeed, when one tries to minimize the distance between the target $T_{i}$ (root only) and the mean shape by summing over the target's points, the optimization process may lead to satisfactory results if $T_{i}$ is bigger than the mean shape root. In this case, the root extremities will match and the upper points of $T_{i}$ will tend toward the upper points of the mean shape root. On the other hand, if $T_{i}$ is smaller, the matching will not work; the upper points of $T_{i}$ will tend toward the closer points of the mean shape root, which do not correspond to the upper part of the mean shape root. Consequently, the root of the reconstructed tooth will be much higher than those of the original model.

\begin{tabular}{l|lll|rll} 
Table IV: Leave-one-test results: reconstruction & \multicolumn{3}{c|}{ HD $(\mathrm{mm})$} & \multicolumn{3}{c}{ HD (\% teeth height) } \\
& Max & Mean & RMS & Max & Mean & RMS \\
\hline Minimum & 0.76 & 0.14 & 0.22 & 3.63 & 0.67 & 1.05 \\
Maximum & 2.86 & 0.82 & 1.11 & 13.68 & 3.92 & 5.31 \\
Mean & 2.01 & 0.41 & 0.57 & 9.61 & 1.96 & 2.73 \\
Variance & 0.5555 & 0.0481 & 0.0901 & 12.7080 & 1.1004 & 2.0612 \\
\hline
\end{tabular}


Table V: Leave-one-test results: reconstruction based on root information (Method 2).

\begin{tabular}{l|lll|cll}
\hline & \multicolumn{3}{|c|}{ HD (mm) } & \multicolumn{3}{c}{ HD (\% teeth height) } \\
& Max & Mean & RMS & Max & Mean & RMS \\
\hline Minimum & 0.88 & 0.14 & 0.19 & 5.14 & 0.82 & 1.11 \\
Maximum & 2.58 & 0.67 & 0.83 & 15.09 & 3.91 & 4.85 \\
Mean & 1.67 & 0.32 & 0.45 & 9.77 & 1.87 & 2.63 \\
Variance & 0.2469 & 0.0175 & 0.0296 & 8.4452 & 0.5986 & 1.0125 \\
\hline
\end{tabular}

Figure 10 shows the Hausdorff distance distribution between $T_{i}$ and $\hat{T}_{i}$ for two different teeth (lighter colors corresponding to larger distances). We notice that even the roots of the target and the mean shape do not always match correctly (cf. tooth on the left). Introducing feature points on both the target and the mean shape can solve this problem. Fig. 11 shows the locations of the feature points. The left image shows the position of the three feature points $F_{1}, F_{2}$ and $F_{3}$ for a given tooth. The right image shows a cross-section of a root and the exact location of $F_{2}$ and $F_{3}$.

These feature points are automatically defined for the two teeth $\left(F_{1, t}, F_{2, t}\right.$ and $F_{3, t}$ for the target and $F_{1, m}, F_{2, m}$ and $F_{3, m}$ for the mean shape). In order to enforce a correct match between the corresponding feature points, a penalty term is then added to the objective function. Eq. (5) is modified to

$$
E(\mathbf{p})=\sum_{i=0}^{M_{c}-1} \min \left(\left\|\mathbf{d}_{j}-\mathbf{m}_{i}\right\|^{2}\right)_{1 \leq j \leq K}+W \sum_{j=1}^{3}\left\|F_{j, t}-F_{j, m}\right\|^{2}
$$

The weight $W$ has to be chosen such that it penalizes the merit function when the feature points are far apart without excessively minimizing the influence of the first term (i.e., the influence of the other points of the target).

Figure 12 shows the effect of adding feature points on the reconstruction. The top three tooth images show the result of the reconstruction without the use of feature points. The three images below correspond to a similar reconstruction process (same number of modes, same algorithm) except that feature points are used.

The use of feature points ensures a better correspondence between the roots of the two 
teeth (original and reconstructed). This leads to a better determination of the crown shape. The different experiments that were carried out showed a slight improvement in the shape determination (the influence is more or less important among the specimens). However, using feature points is not always sufficient to obtain a good approximation of the tooth shape. Modifying Eq. (6) could possibly improve the results further. We also observe that the use of feature points has nearly little effect on the time required for reconstruction of the tooth.

\section{Computation Time}

The algorithm was implemented using MSVC++ and run on a Pentium IV $2.4 \mathrm{GHz}$ personal computer. The computation time related to the the leave-one-out tests described in Sections IV-A and IV-B are summarized in Table VI. For a reconstruction using either crown or root information, Method 2 is much faster than Method 1. Though the distance map allows quick access to the minimum distances, Method 1 is greatly penalized by the computation time of the gradient. Furthermore, Method 1 is more affected by an increase in the density of the statistical model than Method 2 (the running time of Method 1 is proportional to the number of points of the statistical model).

\begin{tabular}{l|cc|cc}
\multicolumn{6}{c}{ Table VI: Computation time for the reconstruction (in seconds). } \\
\hline & \multicolumn{2}{|c}{ Using crown information } & \multicolumn{2}{c}{ Using root information } \\
& Method 1 & Method 2 & Method 1 & Method 2 \\
\hline Minimum & 213 & 20 & 45 & 7 \\
Maximum & 276 & 33 & 60 & 15 \\
Mean & 259.0 & 26.7 & 51.8 & 9.6 \\
\hline
\end{tabular}

\section{Number of Specimens Used}

The results presented above were realized using $N=22$ specimens. To determine if this number was sufficient, we evaluated the influence of the number of specimens used on the reconstruction. Leave-one-out tests (using crown information) were performed using a number 
of specimens $N$ between 2 and 22. For each value of $N, p(p \leq 8)$ groups were constituted randomly. The mean of the mean HD was then evaluated for each value of $N$ and the results are as shown in Fig. 13. This experiment indicates that about 12 teeth is sufficient to obtain a good knowledge about the variability in the tooth type being considered.

\section{E. Number of Modes of Deformation}

Using an optimal or near-optimal number of modes $N_{p c}$ is important as an insufficient number of modes would limit the deformations of our mean model and prevent it taking on the exact shape of the target, whereas a high number of deformation modes would slow down the reconstruction and, above all, introduce undesirable noise. Reconstructions using increasing values for $N_{p c}$ were realized and the results compared. Though not optimal for every reconstruction, the value selected $\left(N_{p c}=7\right)$ leads globally to the optimal results.

\section{CONCLUSiON}

The methods presented here have been proven to be efficient to reconstruct teeth using only crown information and could help clinicians to visualize the outcome of a surgery or choosing between different procedures. Our approach presents two major advantages: it exploits the whole prior information available for a given tooth and it requires little or no interaction from the user.

The reconstructions based on the crown gave a very good precision of the tooth height and width, with an average HD around $10 \%$ between the specimens and their reconstructed shapes. However it gave only a coarse approximation of the teeth shape when using root information only. We have also shown that this approach could be quite fast; indeed, despite the high resolution of our specimens $(105 \mu \mathrm{m})$, Method 2 requires, on average, less than 30 seconds to reconstruct a specimen. 
The method described was developed using a particular kind of tooth. However, it could easily be extended to all single-rooted teeth. Generating a new PDM per tooth is not absolutely necessary; mirror teeth can be reconstructed using the same training set, since the mirror PDM could be created using the mirror view of the mean shape and those of the different modes. Teeth whose shapes differ only in size (e.g., first and second premolar, milk tooth and corresponding adult tooth) could also use the same database by simple scaling of the mean shape and different modes of the PDM.

When 2D information is available in the form of an X-ray and higher accuracy required, combining this information with the proposed method could lead to a better estimation of the PDM's parameters and avoid erroneous reconstructions when dealing with an outlier specimen.

\section{REFERENCES}

[1] Y. Hirogaki, T. Sohmura, H. Satoh, J. Takahashi, and K. Takada, "Complete 3-D reconstruction of dental cast shape using perceptual grouping," IEEE Trans. Med. Imag., vol. 20, no. 10, pp. 1093-1101, Oct. 2001.

[2] M. Alca et al., "An advanced system for the simulation and planning of orthodontic treatment," Medical Image Analysis, vol. 2, no. 1, pp. 61-77, 1998.

[3] R. Cucchiaraa, E. Lammab, and T. Sansoni, "An image analysis approach for automatically re-orienteering CT images for dental implants," Computerized Medical Imaging and Graphics, vol. 28, no. 4, pp. 185-201, June 2004.

[4] N. Noguchi and M. Goto, "Computer simulation system for orthognathic surgery," Orthodontics and Craniofacial Research, vol. 6 (Suppl. 1), pp. 176-178, 2003.

[5] K. Verstreken et al., "An image-guided planning system for endosseous oral implants," IEEE Trans. Med. Imag., vol. 17 , no. 5 , pp. 842-852, Oct. 1998.

[6] D. M. Sarver, Esthetic orthodontics and orthognathic surgery. St. Louis: Mosby, 1998.

[7] S. Keiser-Nielsen, Person identification by means of the teeth. Bristol: John Wright, 1980.

[8] J. Suri, K. Liu, S. Singh, S. Laxminarayana, and L. Reden, "Shape recovery algorithms using level sets in 2-D/3-D medical imagery: A state-of-the-art review," IEEE Trans. in Information Technology in Biomedicine, vol. 6, no. 1, pp. 8-28, Mar. 2002. 
[9] R. Enciso, J. P. Lewis, U. Neumann, and J. Mah, “3D tooth shape from radiographs using thin-plate splines," in "MMVR11 - NextMed: Health Horizon", The 11th Annual Medicine Meets Virtual Reality Conference, Jan. 2003, pp. $62-64$.

[10] R. Poli, G. Coppini, and G. Valli, "Recovery of 3D closed surfaces from sparse data," CGVIP: Image Understanding, vol. 60, no. 1, pp. 1-25, July 1994.

[11] M. Fleute, S. Lavallée, and R. Julliard, "Incorporating a statistically based shape model into a system for computerassisted anterior cruciate ligament surgery," Medical Image Analysis, vol. 3, no. 3, pp. 209-222, 1999.

[12] T. Kondo, S. H. Ong, and K. W. C. Foong, "Tooth segmentation of dental study models using range images," IEEE Trans. Med. Imag., vol. 23, no. 3, pp. 350-362, Mar. 2004.

[13] R. Szeliski and S. Lavallée, "Matching 3D anatomical surfaces with non-rigid deformations using octree-splines," International Journal on Computer Vision, vol. 18, no. 2, pp. 171-186, 1996.

[14] T. W. Sederberg and S. R. Parry, "Free-form deformation of solid geometric models," in ACM Siggraph Conference Proceedings, vol. 20(4), 1986, pp. 151-160.

[15] T. F. Cootes, C. J. Taylor, D. H. Cooper, and J. Graham, "Training models of shapes from sets of examples," in Proceedings of the British Machine Vision Conference, 1992.

[16] L. Lebart, A. Morineau, and M. Piron, Statistique Exploratoire Multidimensionnelle (French), 3rd ed. Dunod, 2000.

[17] W. Lorensen and H. Cline, "Marching cubes: A high resolution 3-D surface construction algorithm," Computer Graphics, vol. 21, pp. 163-169, 1987.

[18] P. J. Besl and N. D. McKay, "A method for registration of 3-D shapes," IEEE Trans. on Pattern Analysis and Machine Intelligence, vol. 14, no. 2, pp. 239-256, Feb. 1992.

[19] W. H. Press, S. A. Teukolsky, W. T. Vetterling, and B. P. Flannery, Numerical Recipes in C++. The Art of Scientific Computing, 2nd ed. Cambridge: Cambridge University Press, 2002.

[20] P. Bhattacharya, "Efficient neighbor finding algorithms in quadtree and octree," Master's thesis, Indian Institute of Technology, Kanpur, Nov. 2001.

[21] A. Moore, "Efficient memory-based learning for robot control," Computer Laboratory, University of Cambridge, Cambridge, UK, Tech. Rep. 209, Oct. 1990.

[22] M. Garland and P. S. Heckbert, "Surface simplification using quadric error metrics," in Proceedings of SIGGRAPH-97, 1997, pp. 209-216. 


\section{List of Figures}

Fig. 1 Fitting of tooth on the dental cast in order to obtain the position of the root.

Fig. 2 Generic model and elastic registration onto a tooth exemplar. (a) Generic model. (b) Tooth exemplar reconstructed for microtomography. (c) Generic model matched onto the exemplar. The mean HD between (b) and (c) equals $0.15 \mathrm{~mm}$.

Fig. 3 (a) Eigenvalues of the autocorrelation matrix in decreasing order. (b) Cumulative percentage of variability given the number of components.

Fig. 4 (a) Influence of the first mode of decomposition on the mean shape. (b) Influence of the second mode of decomposition on the mean shape.

Fig. 5 Octree decomposition realized on a tooth root using 3 levels of decomposition (6 levels are used in the implemented algorithm).

Fig. 6 Examples of reconstruction using crown information only, without the use of a distance map. The 3D mesh represents a given tooth after reconstruction and the surface the original tooth. (a) and (b) are representative specimens while (c) is one particular case among the 22 exemplars.

Fig. 7 Tooth segmentation from a dental cast affects the crown shape: some information is lost on the facial view (left image) and distal view (right image) of the crown extracted.

Fig. 8 Process of fitting a tooth on a dental cast. (a) Crown of the tooth after segmentation. (b) Tooth after reconstruction (gray surface) and original crown (blue surface). (c) Patient's OPG. (d) X-ray of the patient's tooth matched with the tooth reconstructed (blue).

Fig. 9 Examples of reconstruction using root information only, without the use of a distance map. The 3D mesh represents a given tooth after reconstruction and the surface the original tooth. (a) and (b) are representative specimen while (c) is one particular case among the 22 exemplars.

Fig. 10 Distribution of the Hausdorff distance on the reconstructed shapes (reconstruction based on root data). Distances are given in $\mathrm{mm}$.

Fig. 11 Location of the feature points on the root: the image on the left represents the distal view of the root, and the image on the right a $2 \mathrm{D}$ view of the upper part of the root.

Fig. 12 Effect of adding feature points on a reconstruction using root information. Upper row: reconstruction without feature points. Lower row: same tooth reconstructed using three feature points.

Fig. 13 Influence of the number of specimens used to build the statistical model. 


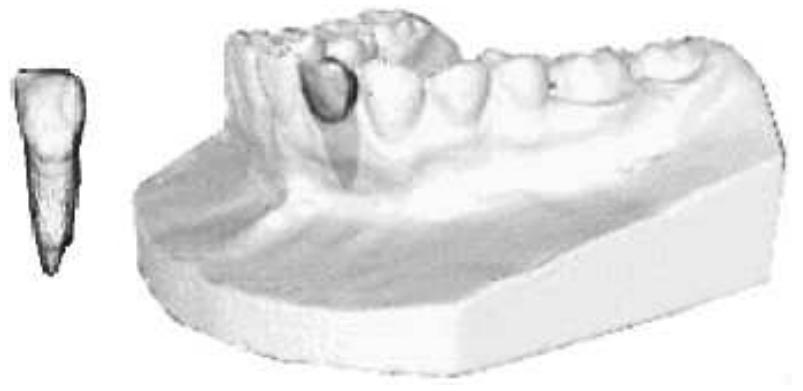

Figure 1 


$$
10
$$




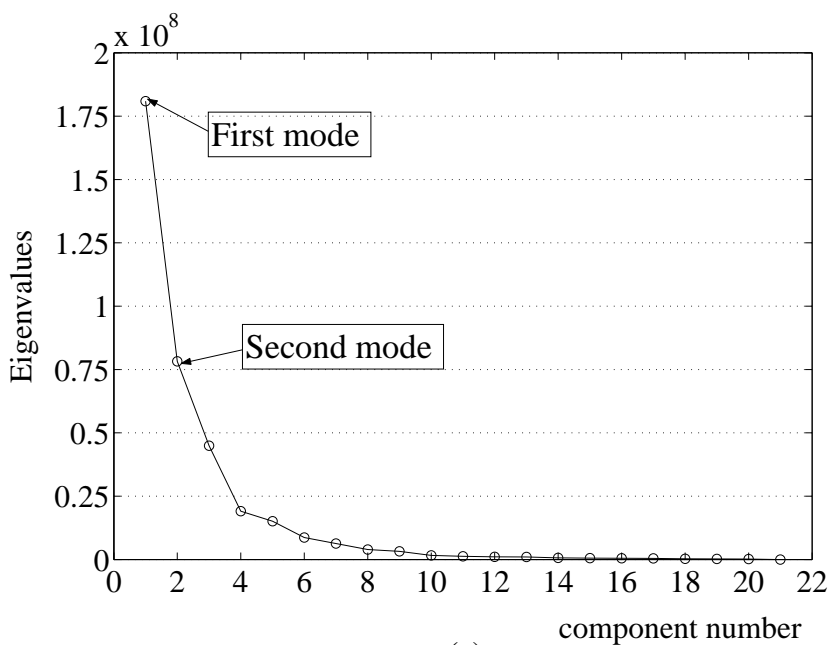

(a)

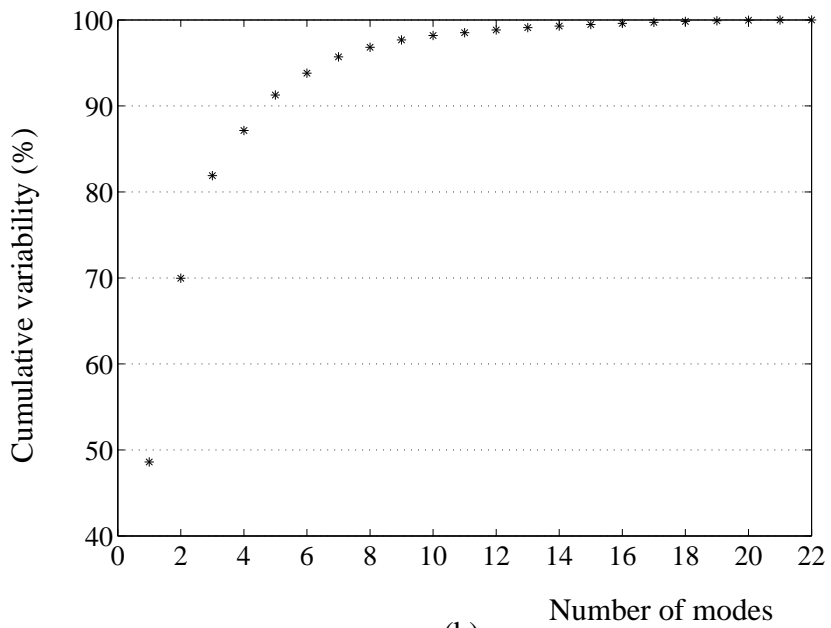

(b)

Figure 3 


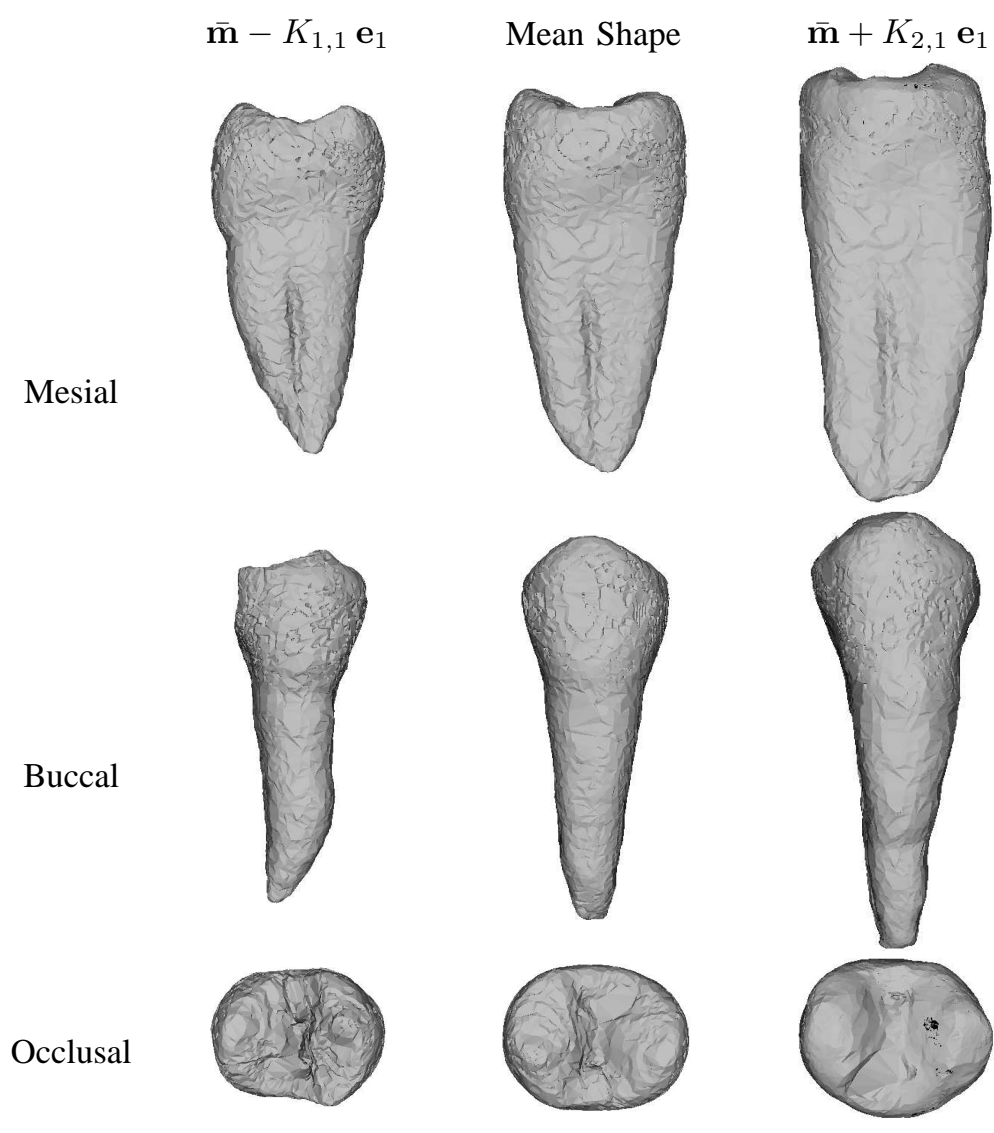

(a)

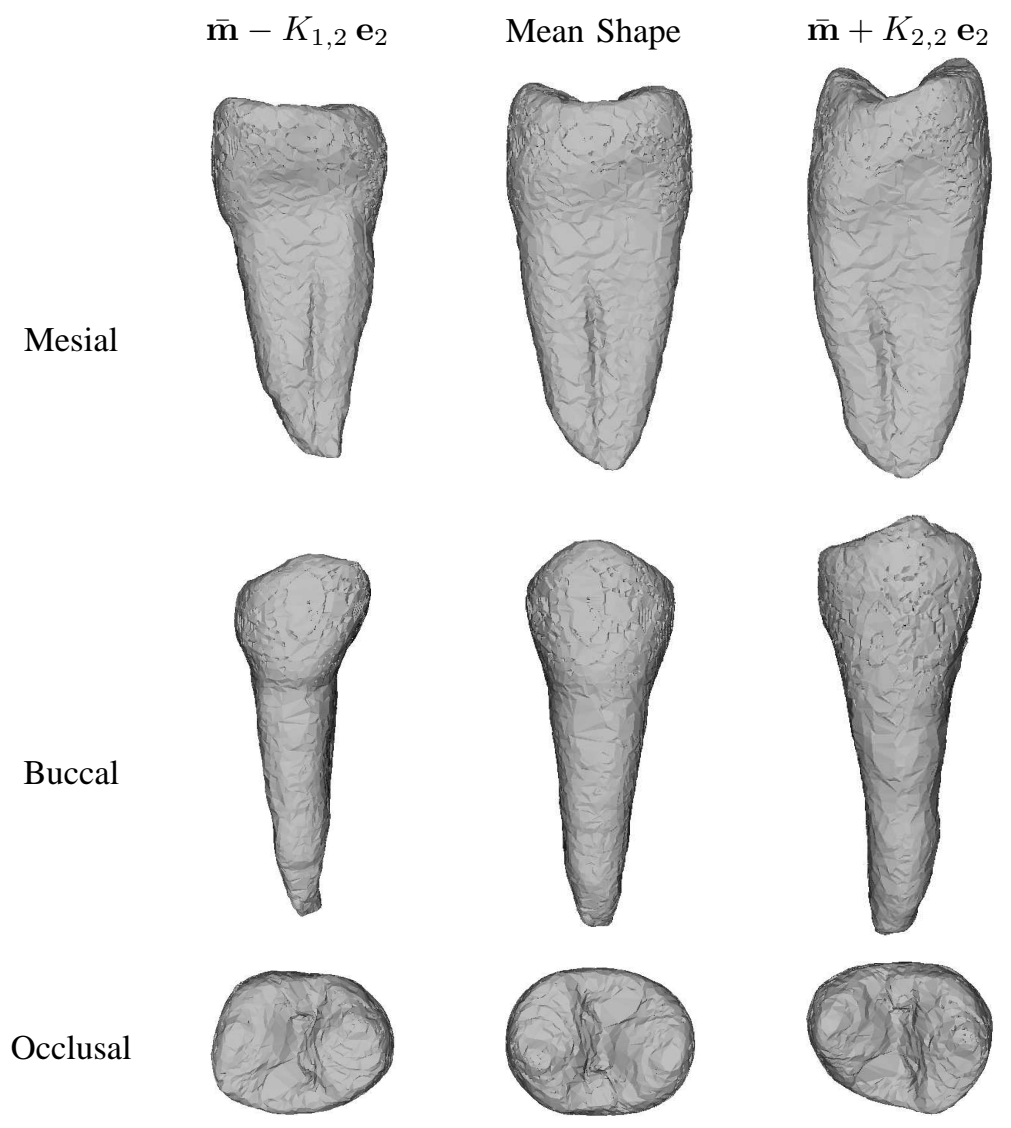

(b)

Figure 4 


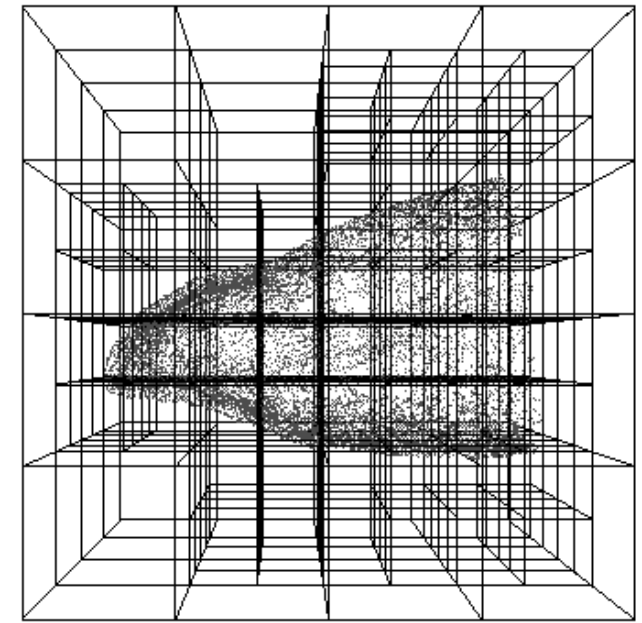

Figure 5 


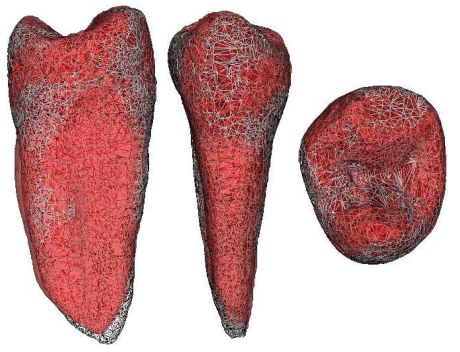

(a)

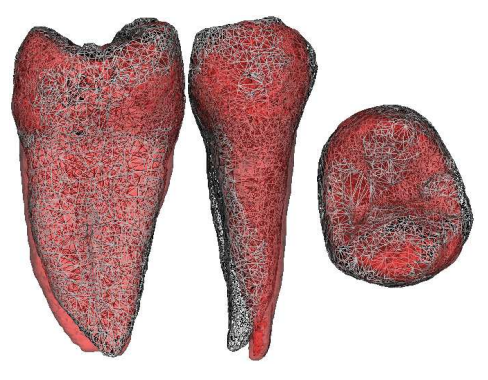

(b)

Figure 6

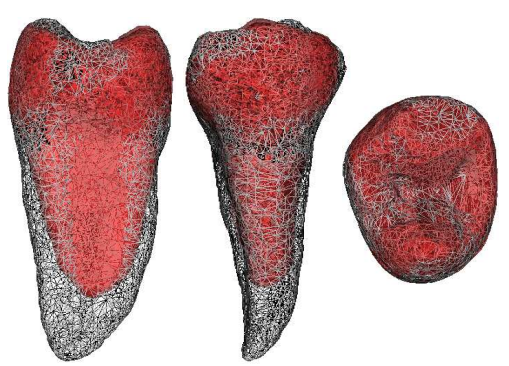

(c) 


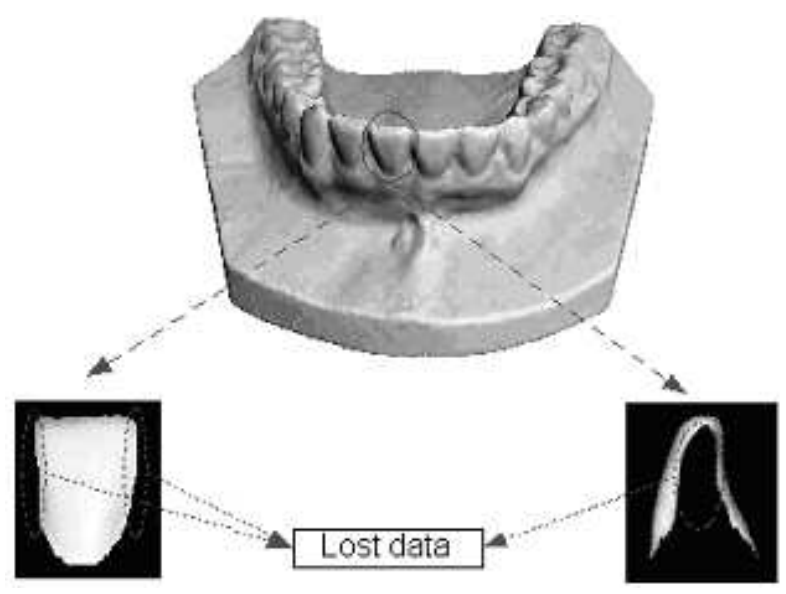

Figure 7 


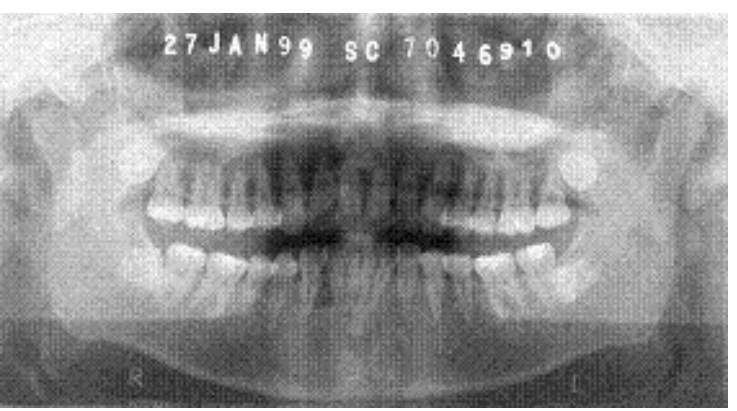

(a)

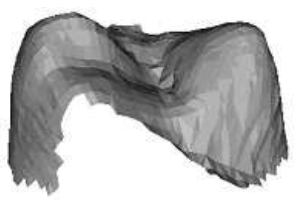

(c)
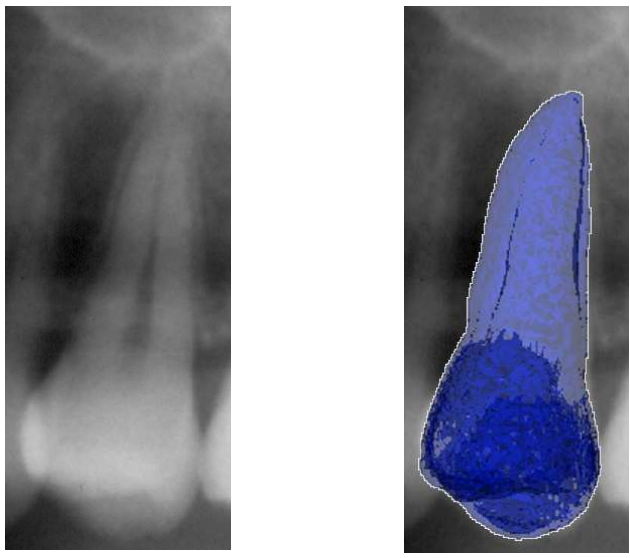

(b)

(d)

Figure 8 


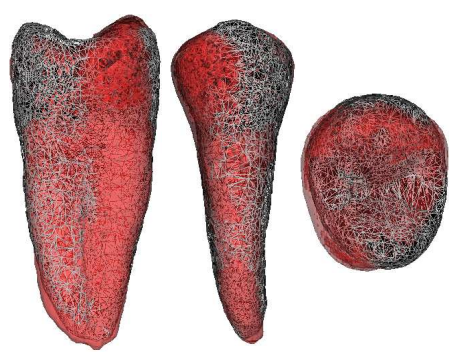

(a)

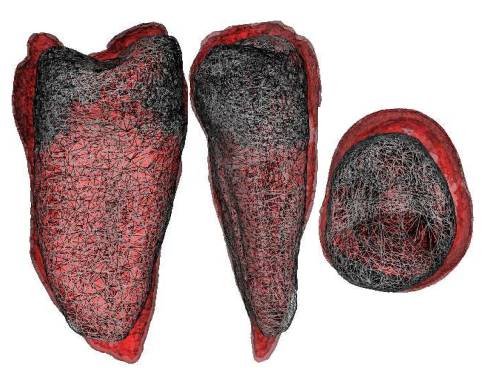

(b)

Figure 9

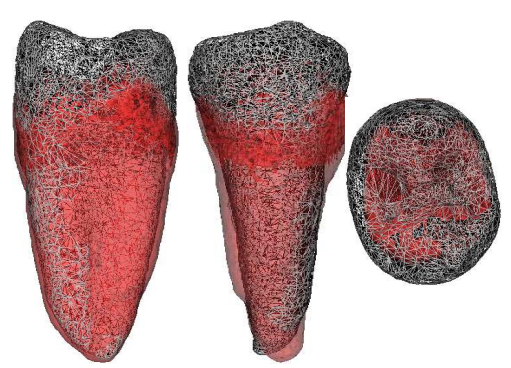

(c) 

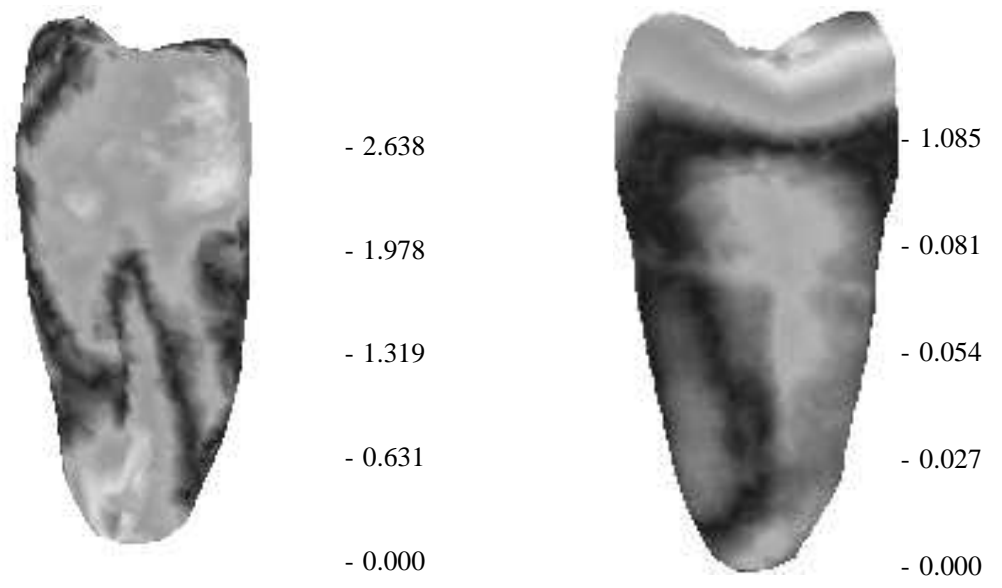

Figure 10 


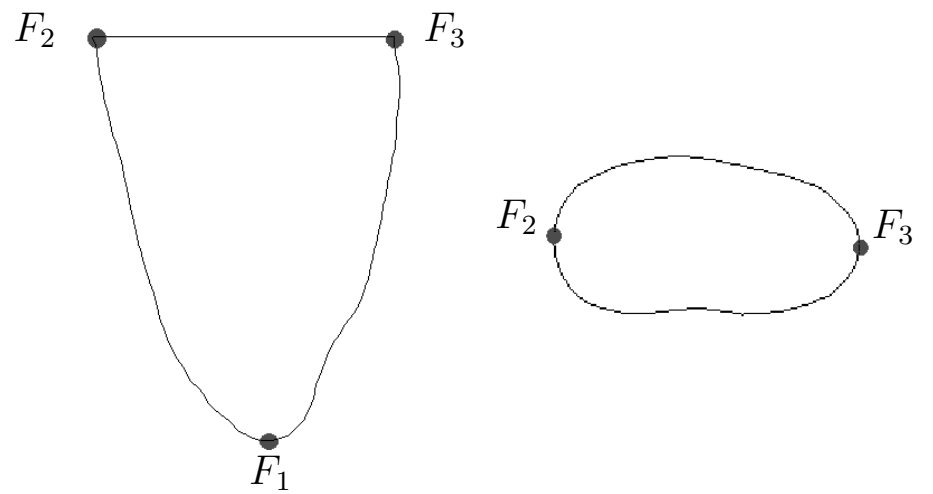

Figure 11 

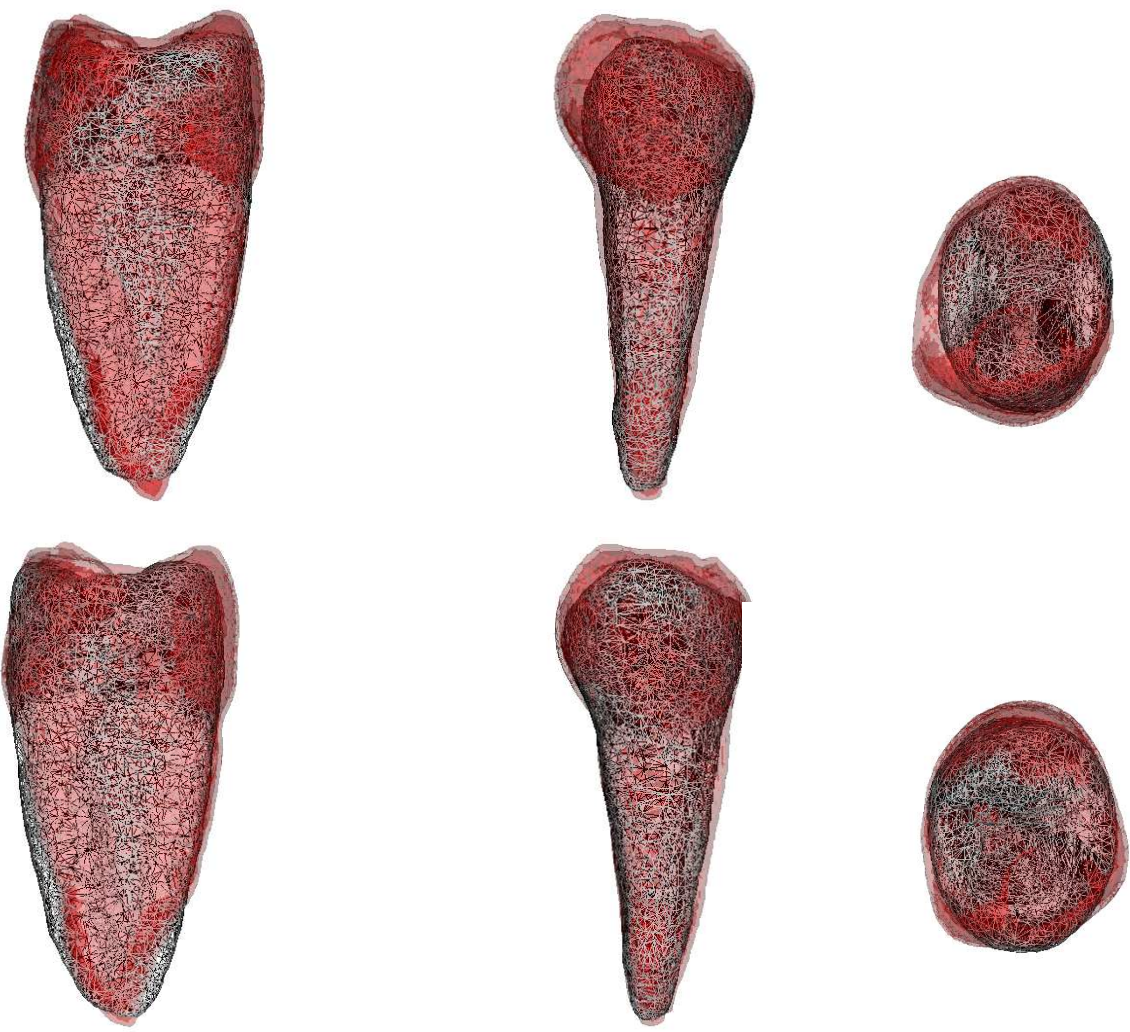

Figure 12 


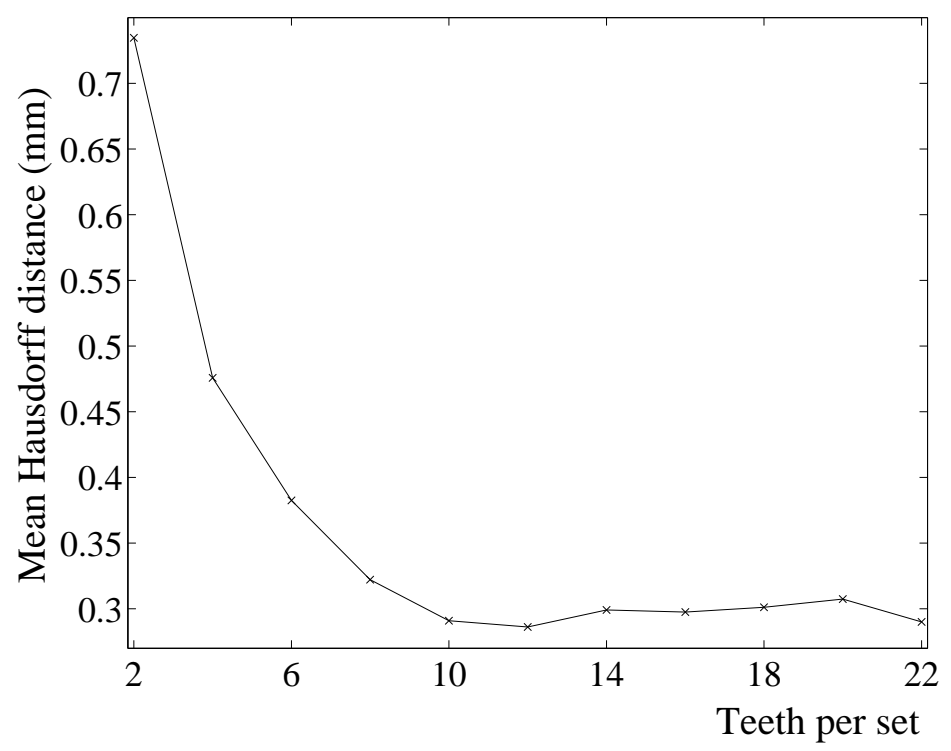

Figure 13 